\title{
Active and Passively Induced Experimental Autoimmune Encephalomyelitis in Common Marmosets: A New Model for Multiple Sclerosis
}

\author{
Luca Massacesi, MD, ${ }^{*}$ Claude P. Genain, MD, ${ }^{*}$ David Lee-Parritz, DVM, $\dagger$ Norman L. Letvin, MD, $\ddagger$ \\ Donald Canfield, DVM, $\dagger$ and Stephen L. Hauser, MD*
}

\begin{abstract}
A chronic relapsing-remitting form of experimental autoimmune encephalomyelitis was induced in the common marmoset Callitbrix jaccbus following a single immunization with human white matter. Individual animals in this species are born as natural bone marrow chimeras, allowing transfer of functional T-cell populations between genetically distinct siblings. The acute disease was characterized clinically by mild neurological signs. Pathologically, the disease was characterized by perivascular mononuclear cell infiltrates, large foci of primary demyelination, and reactive astrogliosis. No animal displayed hemorrhagic-necrotic lesions or polymorphonuclear cell infiltrates characteristic of other acute forms of primate experimental autoimmune encephalomyelitis. A late spontaneous relapse occurred in each of 2 animals followed for 3 to 12 months subsequent to recovery from the acute attack. In these animals, chronic lesions consisted of mononuclear cell infiltrates within large sharply defined areas of demyelination and astrogliosis, and resembled active plaques of chronic multiple sclerosis. Proliferative responses to myelin basic protein but not to myelin proteolipid protein were present in peripheral blood lymphocytes of immunized animals. Furthermore, myelin basic protein-reactive $T$-cell lines derived from immunized donors induced clinical signs of experimental autoimmune encephalomyelitis when adoptively transferred into a sibling, indicating that myelin basic protein-reactive $T$ cells can induce disease in this species. Because of its clinical and pathological similarity to human multiple sclerosis and the ability to adoptively transfer experimental autoimmune encephalomyelitis, this model system should prove useful in the analysis of the immunological mechanisms responsible for autoimmune demyelination in outbred primates.
\end{abstract}

Massacesi L, Genain CP, Lee-Parritz D, Letvin NL, Canfield D, Hauser SL. Active and passively induced experimental autoimmune encephalomyelitis in common marmosets: a new model for multiple sclerosis. Ann Neurol 1995;37:519-530

Experimental autoimmune encephalomyelitis (EAE) describes a group of inflammatory diseases of the central nervous system (CNS) that are induced in susceptible animals by immunization with myelin antigens or by adoptive transfer of sensitized $\mathrm{T}$ cells to syngeneic recipients [1-6]. In inbred rodents, chronic and relapsing-remitting forms of EAE that have been described resemble human multiple sclerosis (MS) [7-9]. More than any other disease model, EAE has contributed to current concepts of T-cell-mediated organ-specific autoimmunity. For example, the understanding of genetic determinants of susceptibility, environmental modifiers, the pathogenic T-cell repertoire, antigen/ epitope recognition, homing, mechanisms of suppression, and cellular contributions of the target organ have been defined in models of EAE [10-15]. EAE has also served in the testing of scores of therapies for MS, yet efficacy has often not been a predictor of benefit in humans. Phylogenetic differences between inbred rodents and outbred humans have probably limited the usefulness of EAE as an MS model.

EAE has been described in macaques, yet acute CNS lesions in these species are hyperacute, hemorrhagic, and destructive, unlike those in MS [16-19]. Chronic $\mathrm{EAE}$ can be induced in some macaques, most notably cynomolgus monkeys, in which the course is characterized by relapses and remissions, by CNS inflammation, demyelination, and gliosis, and by $\mathrm{T}$-cell responses to myelin basic protein (MBP) that correlate with the development of clinical signs $[20,21]$. EAE has also been
From the *University of California, San Francisco, Department of Neurology, San Francisco, CA; †The New England Regional Primate Research Center, Southborough, MA; and $\$$ Harvard Medical School, Beth Israel Hospital, Boston, MA.
Received Jun 29, 1994, and in revised form Oct 13. Accepted for publication Nov 22, 1994.

Address correspondence to Dr Hauser, Department of Neurology, University of California, San Francisco, Box 0114, San Francisco, CA 94143 . 
described in owl and squirrel monkeys [22-24]. The outbred nature of nonhuman primates has limited their value as disease models, since adoptive transfer of genetically compatible $\mathrm{T}$ cells between animals is valuable for elucidating the role of specific $\mathrm{T}$-cell populations in EAE. To date, no practical model of an MS-like lesion has been described in nonhuman primates.

Here we describe the induction and the characteristics of EAE in the common marmoset Callitbrix jacchus. These are small New World monkeys (weighing in adulthood $300-400 \mathrm{gm}$ ), whose care and handling in captivity is relatively simple. Actively induced EAE in $C$. jacchus is characterized clinically by mild neurological signs and a relapsing-remitting course, and pathologically by mononuclear cell infiltration, primary demyelination, and reactive gliosis. A further advantage for the use of common marmosets as a model for EAE is that they are born as naturally occurring bone marrow chimeras [25]. While individual animals from multiple births arise from separate ova that are fertilized independently, the placentae of the developing animals fuse, resulting in a cross circulation of bone marrow-derived elements between the developing fetuses. Thus, while the animals are genetically distinct, they share and are tolerant of each other's bone marrow-derived cell populations. We now demonstrate that it is possible to adoptively transfer EAE by T-cell transfer between members of a chimeric set of twins. Acute and chronic EAE, created in a species whose immune and nervous system genes are phylogenetically close to those of humans, represents a unique disease model that should prove useful in elucidating immune mechanisms of CNS demyelination.

\section{Materials and Methods \\ Animals \\ C. jaccbus animals were maintained in primate colonies at the University of California, San Francisco (UCSF) and the New England Regional Primate Research Center. The animals used in this study were cared for in accordance with the guidelines of the Committee on Animals of the Harvard Medical School and UCSF, and those of the Committee on Care and Use of Laboratory Animals of the Institute of Labo- ratory Animal Resources, National Research Council. A maximum of $2.5 \mathrm{ml}$ of blood every other week was taken from each animal.}

\section{Induction of Experimental Autoimmune Encephalomyelitis}

Nine animals were immunized with $200 \mathrm{mg}$ of fresh-frozen postmortem human brain white matter homogenate $(\mathrm{BH})$ emulsified with complete Freund's adjuvant (CFA) containing $3 \mathrm{mg} / \mathrm{ml}$ of killed Mycobacterium tuberculosis (H37 Ra strain). Intradermal injections (tocal volume of $0.6 \mathrm{ml} /$ animal) were divided in four sites on the dorsal axilla and inguinal region. On the day of immunization and again 2 days later, $10^{10}$ inactivated Bordetella pertussis organisms (Bordetella per- tussis vaccine, Massachusetts Public Health Department Biological Laboratories, Boston, MA) were diluted in $10 \mathrm{ml}$ of saline solution and administered by the intravenous route. In animals that developed fever due to the initial pertussis vaccine, the second pertussis injection was delayed by 1 to 2 days. Two to 5 weeks after immunization, some animals developed local skin ulcerations related to the CFA. In these animals, cephalexin ( $20 \mathrm{mg} / \mathrm{kg}$ twice daily for 10 days) was administered intramuscularly to prevent secondary infection.

EAE was assessed by clinical and pathological criteria. A standardized scoring system was employed to record the severity of clinical disease: $0=$ normal neurological findings; 1 = lethargy, anorexia, weight loss; 2 = ataxia, and either paraparesis/monoparesis, sensory loss, or brainstem syndrome including gaze palsy, or blindness; 3 = paraplegia or hemiplegia; 4 = quadriplegia.

Animals were killed at different times after immunization. The CNS was removed and fixed in $10 \%$ formalin. Paraffin sections of brain and spinal cord were prepared and stained with hematoxylin and eosin, or with Luxol fast blue. For each coronal brain section or horizontal spinal cord section, the histopathological findings of inflammation and demyelination were each independently graded according to an arbitrary scale:

Inflammation Score

$0 \quad$ No inflammation present

$+\quad$ Rare (1-3) perivascular cuffs/average whole section

++ Moderate numbers (3-10) of perivascular cuffs/section; may have meningeal inflammation

+++ Widespread perivascular cuffing and parenchymal infiltration by inflammatory cells

\section{Demyelination Score}

$0 \quad$ No demyelination present

$1+$ Rare (1-3 lesions/section) foci of demyelination

$2+$ Moderate (3-10 lesions/section) demyelination

$3+$ Extensive demyelination with large confluent lesions

\section{Proliferation Studies}

Under sterile conditions, spleen and lymph node tissue was harvested and single cell suspensions prepared by passage through a stainless-steel mesh. Peripheral blood mononuclear cells (PBMCs) were prepared from heparinized venous blood within 3 hours of venipuncture by density gradient centrifugation using Ficoll-Hypaque. Spleen cells, lymph node cells, or PBMCs $\left(2 \times 10^{5} /\right.$ well $)$ were cultured in a total volume of $200 \mu \mathrm{l}$ in round-bottom microtiter plates (Falcon Plastics, Oxnard, CA) in culture medium consisting of RPMI 1640 supplemented with $10 \%$ controlled processed serum replacement-2 (Sigma Pharmaceuticals, St. Louis, MO), 2 $\mathrm{mM}$ I-glutamine (Gibco Laboratories, Grand Island, NY), $20 \mathrm{mM}$ 4-(2-hydroxyethyl)-1-piperazine ethane-sulfonic acid (HEPES) buffer (Sigma), and penicillin-streptomycin (Gibco). To some wells the following antigens (final concentration) were added:

A. Human MBP $(50 \mu \mathrm{g} / \mathrm{ml})$ prepared by the method of Deibler and colleagues [26].

B. Human proteolipid protein (PLP) prepared from a human 
brain total lipid extract [27]; lipid was removed by chromatography on a Sephadex LH 60 column eluted with chloroform:methanol:acetic acid [28]. The eluate was converted to the water-soluble form as described by Lees and Sakura [29].

C. Human $\mathrm{BH}, 0.1 \%$, sonicated and irradiated.

After 4 days of incubation, $\left[{ }^{3} \mathrm{H}\right]$ thymidine $(1 \mu \mathrm{Ci} /$ well $)$ was added, cells were harvested 16 hours later, and thymidine incorporation was measured in a scintillation counter. Proliferative responses were expressed as stimulation indices (SIs), defined as the stimulated cultures (counts per minute) in unstimulated cultures in triplicate wells.

\section{Adoptive Transfer Experiments}

SHORT-TERM LINES. Following onset of neurological signs of EAE, the animals were sacrificed and the spleen and draining lymph nodes were aseptically removed. Washed lymph node $\left(3 \times 10^{6} / \mathrm{ml}\right)$ and spleen $\left(10^{6} / \mathrm{ml}\right)$ cells were cultured together in the presence of human MBP $(50 \mu \mathrm{g} / \mathrm{ml})$ in complete medium (RPMI 1640 containing 10\% controlled processed serum replacement-2) (Fig 1). After 4 days the responding cell population was enriched by Ficoll-Hypaque density gradient centrifugation, washed, and resuspended in growth medium, that is, complete medium containing $5 \%$ human T-cell growth factor (TCGF) (Cellular Products), 40 units/ml of human recombinant interleukin (IL)-2 (Hazelton), and 4 units/ml of human recombinant IL-4 (Genzyme). After 12 days in growth medium, the cells were washed, restimulated with MBP for 3 days in the presence of equal numbers of syngeneic spleen cells (frozen prior to use) as antigen-presenting cells (APCs), cultured in growth medium for 1 additional day, and washed. Activated cells were again enriched by density gradient centrifugation and washed, and $40 \times 10^{6}$ cells were resuspended in $5 \mathrm{ml}$ of phosphate-buffered saline solution (PBS) and injected intravenously into the naive (unimmunized) sibling (see Fig 1). Each transferred cell line was subsequently expanded in vitro by alternating cycles of stimulation with MBP and growth medium for epitope mapping studies.

LONG-TERM LINES. Long-term MBP-reactive T-cell lines were derived by limiting dilution. Mixtures of splenic and lymph node cells (ratio of $2: 1$ ) were cultured at $10^{5}$ cells/ well in 96 round-bottom well plates with purified human MBP $(50 \mu \mathrm{g} / \mathrm{ml}$ final concentration) in complete medium. After 3 days the culture medium was supplemented with growth medium and this medium was then changed every 3 days for 9 days. $T$ cells were restimulated at the end of the cycle by addition of $2 \times 10^{5}$ freshly isolated chimeric PBMCs pulsed with MBP ( $50 \mu \mathrm{g} / \mathrm{ml}$ ) and irradiated (30 Gy) as APCs. Lines were expanded by multiple cycles of restimulation with MBP/APCs followed by culture in growth medium.

At the end of the stimulation cycles, $10^{7} \mathrm{~T}$ cells were rested for 48 hours in controlled processed serum replacement- 2 medium, then restimulated with $\operatorname{MBP}(50 \mu \mathrm{g} / \mathrm{ml})$ in the presence of an equal number of irradiated $(30 \mathrm{~Gy})$ freshly isolated PBMCs as APCs. Growth medium was added after 48 hours, and 24 hours later cells were harvested, washed

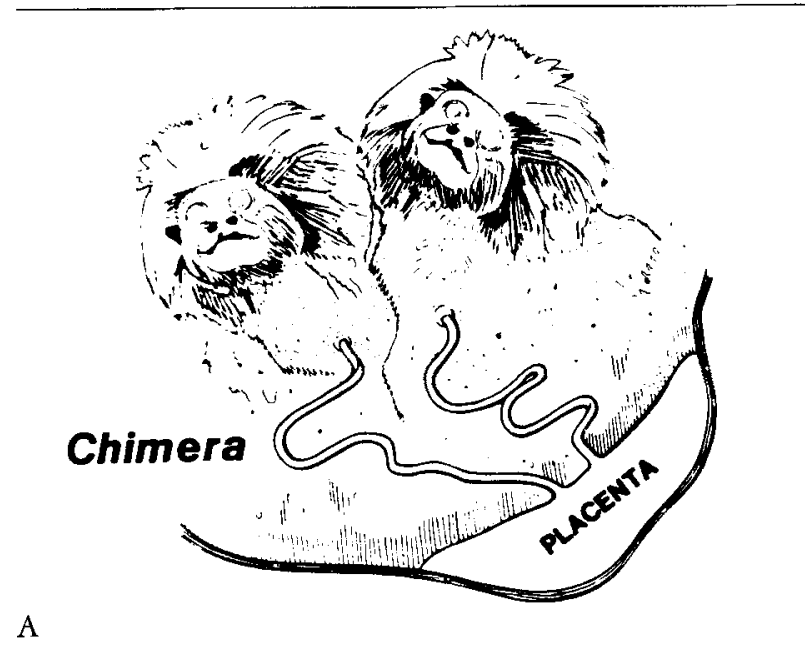

A

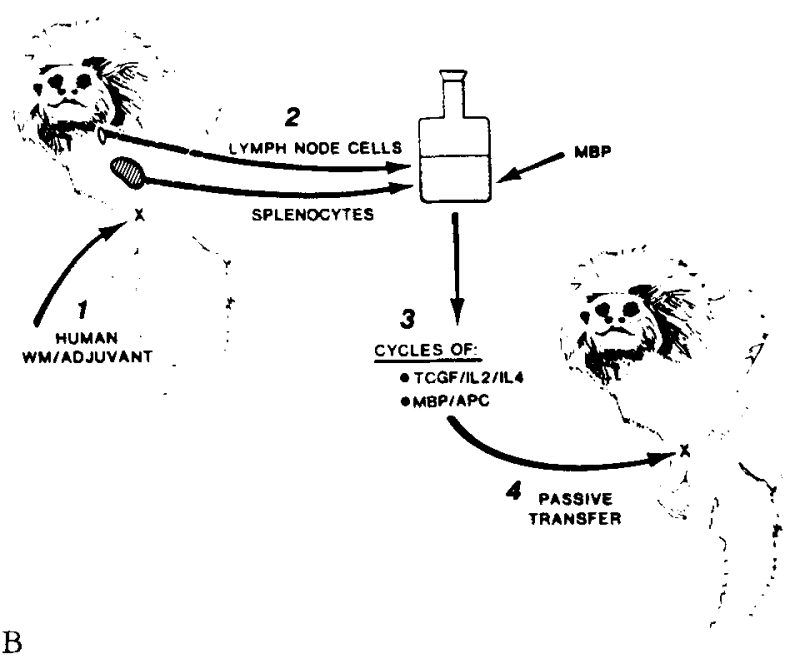

Fig 1. (A) The natural chimerism of Callithrix jacchus. Animals typically develop as genetically distinct fetuses that share a common fetal blood supply. This creates a permanent and stable bone marrow chimeric state. (B) Metbod for induction of adoptive transfer of experimental autoimmune encephalomyelitis between chimeric siblings mediated by myelin basic protein (MBP)-reactive $T$-cell lines. $W M=$ white matter; $I L=i n$ terleukin; $T C G F=T$-cell growth factor; $A P C=$ antigenpresenting cell.

with RPMI, resuspended in $2 \mathrm{ml}$ of $0.9 \%$ saline solution, and injected intravenously into the recipient.

All recipient animals received intravenous injections of $10^{10}$ Bordetella pertussis on the day of transfer and again 48 hours later.

Lines were screened for fine antigen specificity using a panel of 20-mer overlapping peptides corresponding to the amino acid sequence of human MBP. Human and monkey MBP differ by only 4 of 172 amino acids (aa) [30]. Proliferative responses (SIs) were measured as described above, using $5 \times 10^{4} \mathrm{~T}$ cells stimulated with $10^{5}$ irradiated ( $30 \mathrm{~Gy}$ ) frozen autologous spleen cells or freshly isolated chimeric PBMCs, in the presence of $100 \mu \mathrm{g} / \mathrm{ml}$ of each MBP peptide. 


\section{Results \\ Experimental Autoimmune Encephalomyelitis Induced by Active Immunization in $\mathrm{C}$. jacchus Monkeys}

Signs of EAE developed in all 9 animals immunized with white matter homogenate. The onset of the initial illness ranged from day 7 to day 30 following immunization. Commonly present were signs of ascending paraparesis or paralysis, tremor, loss of pain sensation in the tail or hind legs, and blindness. The initial clinical deficit stabilized rapidly, within 24 hours from the onset, at a median score of 2.0. No C. jacchus had an initial score greater than 3 (Table).

Three animals (Animals 96-89, 183-88, and 130-88) were not sacrificed at disease onset and were followed for 3, 6, and 18 months, respectively. In each animal, complete clinical recovery from the initial attack occurred between 1 and 4 months after immunization (Fig 2). Two animals were followed without additional intervention, and spontaneous relapses occurred in both. In the first, a relapse occurred on day 180 and persisted 4 months and remitted without residual deficits (Fig 2A). In the second, a spontaneous relapse on day 110 resulted in severe quadriplegia, necessitating euthanasia (Fig 2B). A third animal was actively reimmunized following recovery, and recurrent disease developed 27 days later. This suggests that remission was not associated with resistance to reinduction (Fig 2C), in contrast to most rodent models of $\operatorname{EAE}[31,32]$. Based on this single observation we could not exclude the possibility that a "spontaneous" relapse that was unrelated to the reimmunization occurred.

\section{Patbology}

In acute EAE, the spinal cord appeared macroscopically soft and swollen. On microscopic examination, acute lesions were multiple, perivascular in location, and with rare exceptions, restricted to white matter.
Occasional lesions were noted at gray-white matter junctions. Although lesions were present throughout the neuroaxis, spinal cord lesions were particularly prominent. Involvement of the cord was relatively symmetrical and predominated in posterior and lateral funiculi (Figs 3A-3D). Perivascular cellular infiltrates were comprised exclusively of mononuclear cells (Figs $3 E, 3 F$ ). Plasma cells were rare. In no lesions were polymorphonuclear cells, hemorrhage, or necrosis observed. The most remarkable feature of the acute lesion was the large size and the sharply delimited areas of primary demyelination that were associated with cellular infiltrates. These areas were densely packed with infiltrating macrophages containing myelin debris, and were associated with early reactive gliosis.

Animals 183-88, 96-89, and 130-88 were sacrificed, respectively, 3, 6, and 18 months after the onset of neurological signs. In each, inflammatory infiltrates were present, located not only in perivascular locations but also diffusely in white matter parenchyma corresponding to foci of demyelination and dense reactive gliosis (Fig 4). Little meningeal or subpial inflammation was present (see Figs 4A, 4B). Within infiltrates, most cells had the morphological appearance of monocytes or macrophages. Lymphocytes also were present in chronic lesions, located in a perivenular distribution or at the lesion edge. The characteristics of these round or oval, sharply demarcated chronic lesions (i.e., mononuclear cell infiltrates, demyelination, and gliosis centered around a venule) were indistinguishable morphologically from plaques observed in human MS (see Figs $4 \mathrm{E}, 4 \mathrm{~F})$.

\section{Lymphocyte Proliferative Responses}

Proliferative responses to $\mathrm{BH}, \mathrm{MBP}$, and PLP were measured from PBMCs prior to immunization and from PBMCs, spleen cells, and lymph node cells at the

Clinical and Histopatbological Findings in Experimental Autoimmune Encepbalomyelitis Induced in Callithrix jacchus Monkeys

\begin{tabular}{|c|c|c|c|c|c|}
\hline \multirow[b]{2}{*}{ Animal No. } & \multirow[b]{2}{*}{ Time of Onset ${ }^{2}$} & \multirow[b]{2}{*}{ Maximum Score ${ }^{b}$} & \multirow{2}{*}{$\begin{array}{l}\text { Score (Day) } \\
\text { at Time of Deaths }{ }^{\mathrm{a}}\end{array}$} & \multicolumn{2}{|c|}{ Pathology $y^{\mathrm{c}}$} \\
\hline & & & & Inflammation & Demyelination \\
\hline $130-88$ & 30,180 & 3,2 & $0(550)$ & ++ & ++ \\
\hline $131-88$ & 23 & 3 & $3(25)$ & ++ & +++ \\
\hline $183-88 a$ & 24 & 2 & (ND) & ND & $\mathrm{ND}$ \\
\hline $183-88 b^{d}$ & 155 & 3 & $3(188)$ & ++ & +++ \\
\hline $367-88$ & 29 & 2 & $2(33)$ & +++ & +++ \\
\hline $116-89$ & 18 & 2 & $2(25)$ & ++ & $++e$ \\
\hline $344-89$ & 14 & 2 & $2(40)$ & ++ & $++^{e}$ \\
\hline $54-90$ & 16 & 2 & (ND) & ND & ND \\
\hline $48-90$ & 16 & 2 & (ND) & ND & ND \\
\hline $96-89$ & 7,110 & 2,4 & $4(114)$ & +++ & $++t$ \\
\hline
\end{tabular}

${ }^{2}$ Days after immunization.

${ }^{b}$ Clinical scoring system described in Materials and Methods.

'See Materials and Methods for explanation of grading system.

${ }^{d}$ Animal 183-88 was reimmunized at day 128 after first immunization.

'Spinal cord abnormalities were less severe in Animals 116-89 and 344-89 than in the other animals examined.

$\mathrm{ND}=$ not done. 

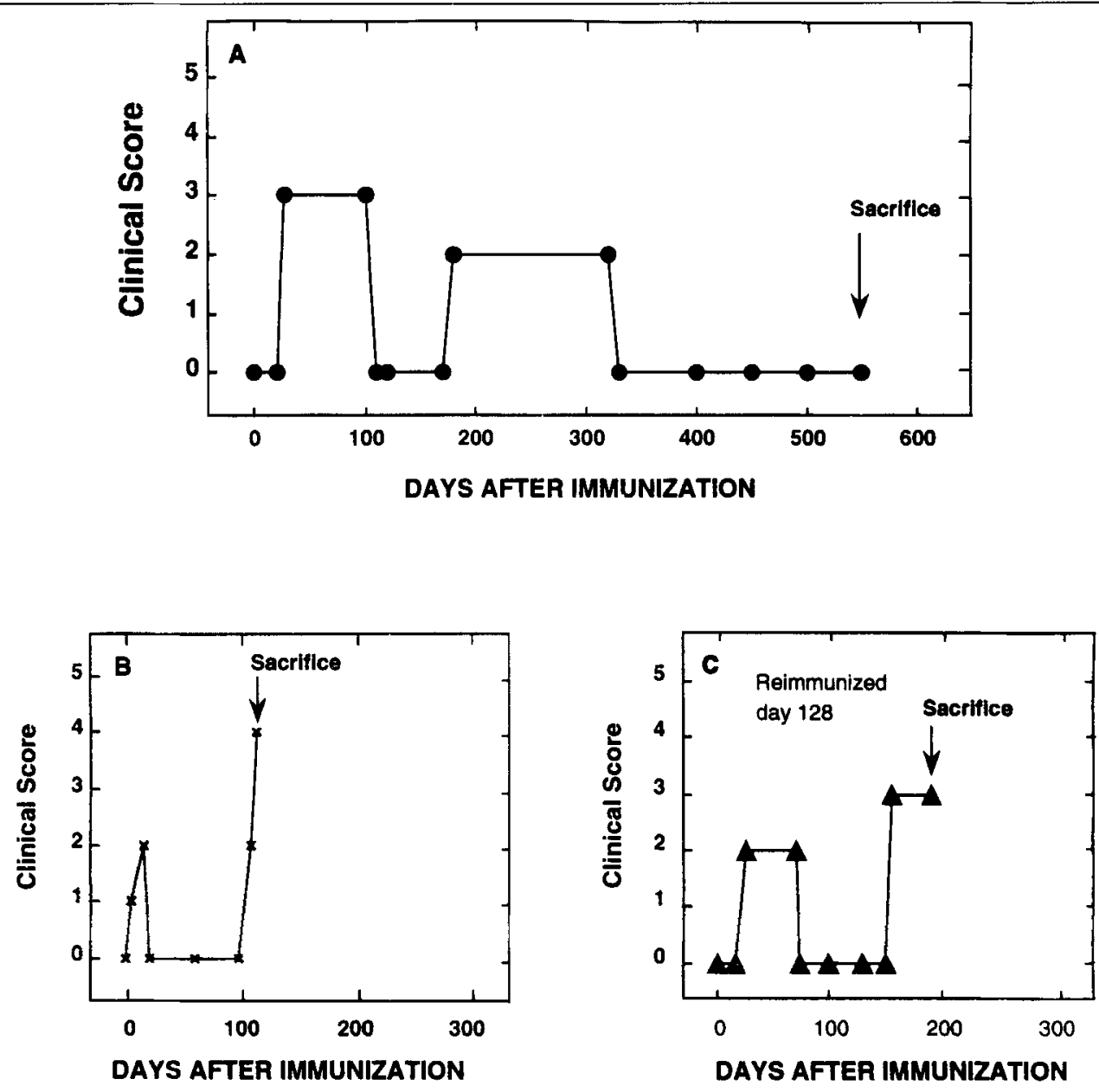

Fig 2. The clinical course of experimental autoimmune encepbalomyelitis in 3 representative animals. (A) Animal 130-88. (B) Animal 96-89. (C) Animal 183-88.

time of death. From PBMCs, a proliferative response to both $\mathrm{BH}$ and $\mathrm{MBP}$ was present in most animals (Fig 5). Specific responses of spleen and lymph node cells to $\mathrm{MBP}$ and $\mathrm{BH}$ were also present in most animals at the time of death. By contrast, proliferative responses to PLP could not be detected in most animals studied (see Fig 5). These data suggest that in acute EAE, responses to myelin antigens that are detectable in peripheral blood reflect to some degree those present in lymphoid tissue. Furthermore, they indicate that a specific T-cell response to MBP was present in affected animals.

\section{Adoptive Transfer Experimental Autoimmune Encephalomyelitis}

In total, four adoprive transfers were performed. In two transfers that employed short-term MBP-reactive T-cell lines, clinical signs of EAE developed in both recipients. In the first, acure paraparesis was followed

by complete clinical recovery lasting more than 2 months (Fig 6A). In the second, a relapsing-remitting disease followed adoptive transfer (Fig 6B).

Two other transfers employed long-term MBPreactive $\mathrm{T}$-cell lines. One line (17.i5.2.28, $\mathrm{SI}=16$, specificity aa 31-50) induced paraparesis and sensory loss in the recipient 8 days following transfer (Fig 6C). This animal partially recovered but then one additional spontaneous relapse developed during a 1-year period of additional observation. A second MBP-reactive line (21.C.2.100, SI = 21, specificity aa 92-111) failed to induce clinical disease when the recipient animal was observed for 120 days following adoptive transfer.

Disease severity was milder in EAE mediated by adoptive transfer of $\mathrm{T}$-cell lines (maximum score of 2.0 in each recipient) than in EAE induced by active immunization with $\mathrm{BH} / \mathrm{CFA}$. Adoptive transfer recipients were sacrificed 3 to 12 months following the initial induction of EAE. In each of these 3 recipients, postmortem examination was performed. In 2 transfer recipients, no abnormalities of significance were found, and in the third only a single necrotic focus of uncer- 


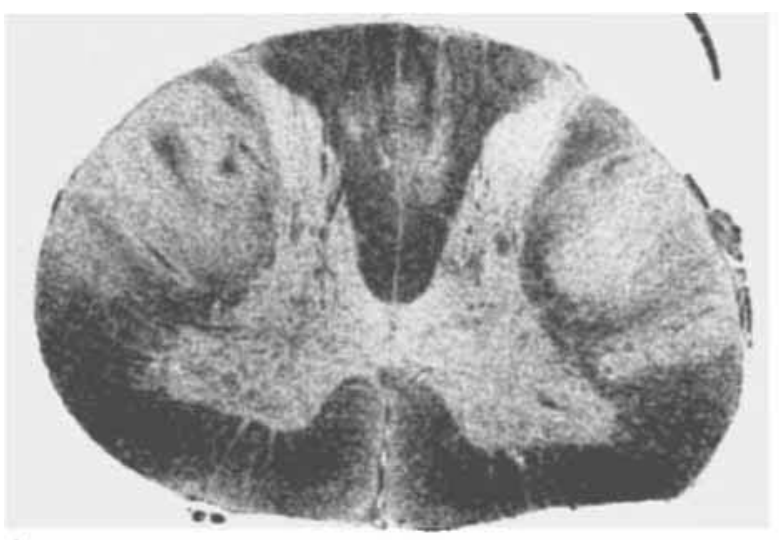

A

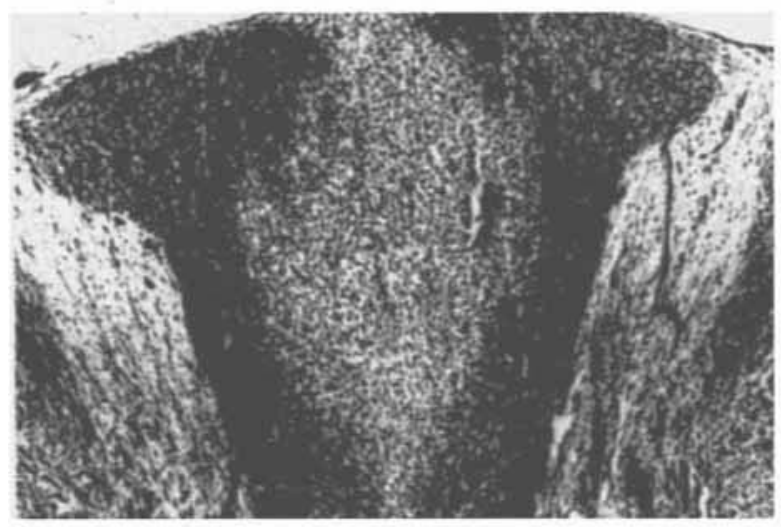

C

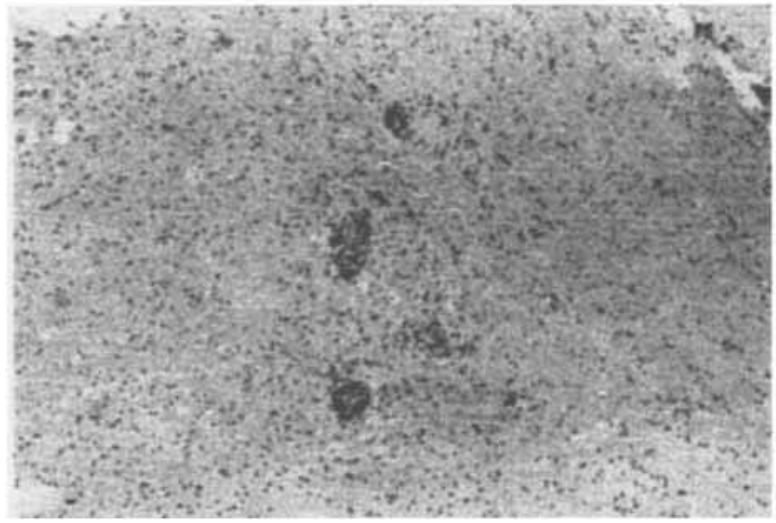

E

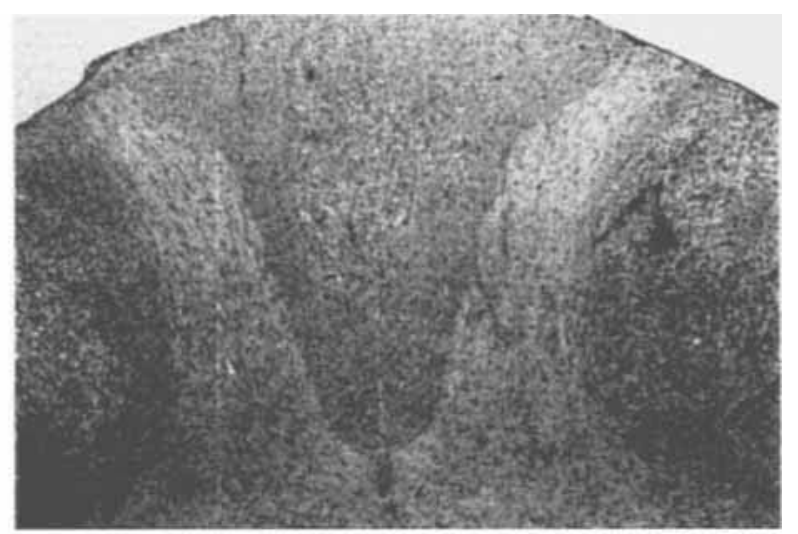

B

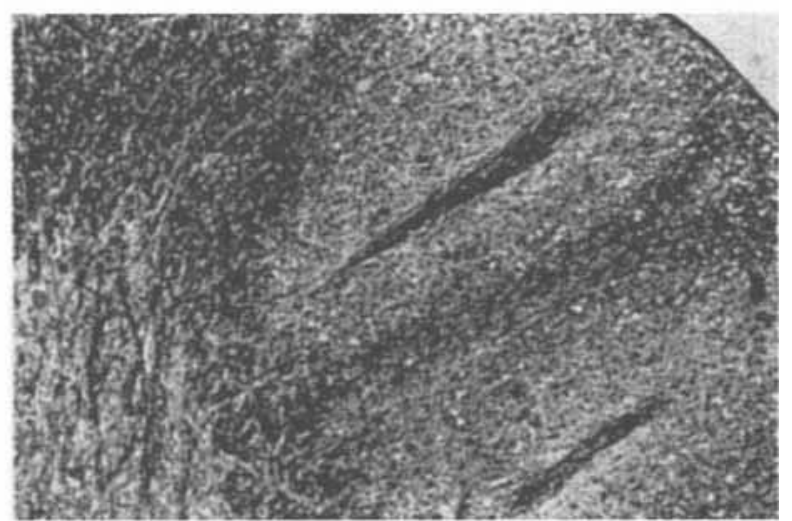

D

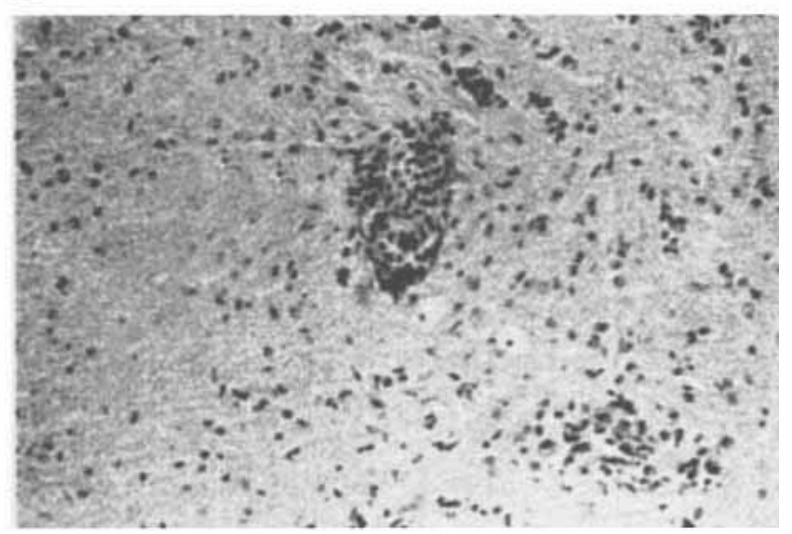

F
Fig 3. Patbological features of acute experimental autoimmune encepbalomyelitis in $\mathrm{C}$. jacchus. (A-D) Horizontal thoracic spinal cord sections (Animal 131-88) illustrate perivascular inflammation, macrophage inflitration, and large demyelinated lesions in the lateral and posterior funiculi. (A: Hematoxylin and eosin (HEE), $\times 50$ before $9 \%$ reduction; B: HEE, $\times 100$ before $35 \%$ reduction.) (C) Large demyelinating lesion within the posterior columns; note the extensive demyelination with sharply defined edges and dense macrophage infiltration. (Luxol fast blue/periodic acid-Schiff stain, $\times 160$ before $36 \%$ reduction.) (D) In the lateral columns, perivascular cuffing and surrounding demyelination. ( $\times 150$ before $36 \%$ reduction.) (E, F) A section through the corona radiata at the level of the caudate putamen (Animal 367-88) illustrates a multifocal perivascular infiltrate and early astrogliosis. $(E: \times 200$ before $36 \%$ reduction; $F: \times 500$ before $36 \%$ reduction.) 


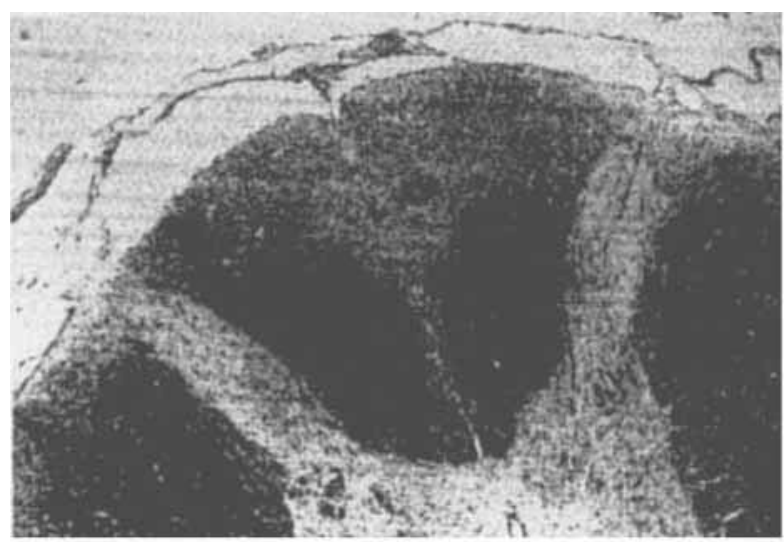

A

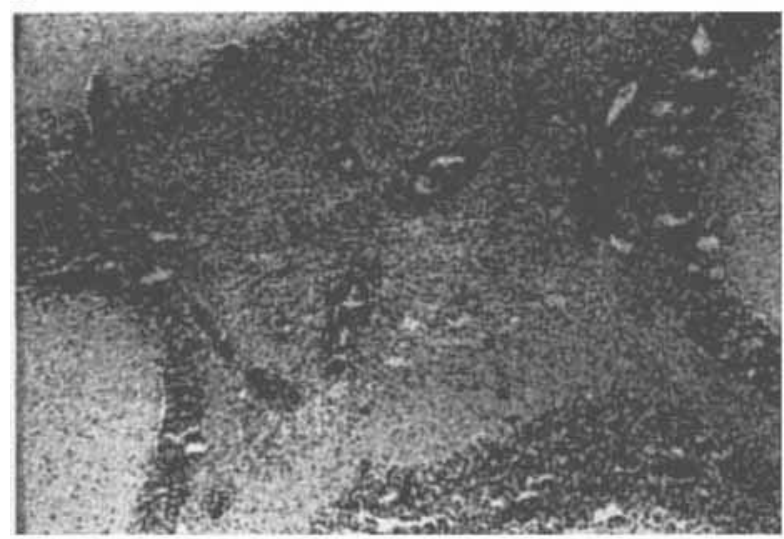

C

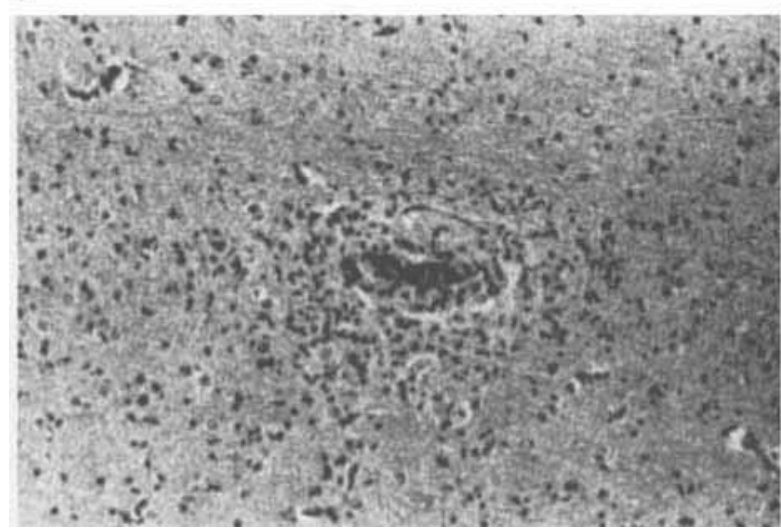

E

Fig 4. Patbological features of chronic experimental autoimmune encepbalomyelitis in C. jacchus. (A) Thoracic spinal cord (Animal 130-88). Confuent perivascular and subpial demyelination in the posterior funiculi. (Luxol fast bluelperiodic acid-Schiff stain. $\times 100$ before $34 \%$ reduction.) (B) Dorsal pons adjacent to the fourth ventricle. Multifocal perivenular inflitrates. (Hematoxylin and eosin (HEE), $\times 133$ before $35 \%$ reduction.) (C) Cerebellum (Animal 183-88). Large demyelinating lesion with densely packed macrophages and multiple perivascular in-

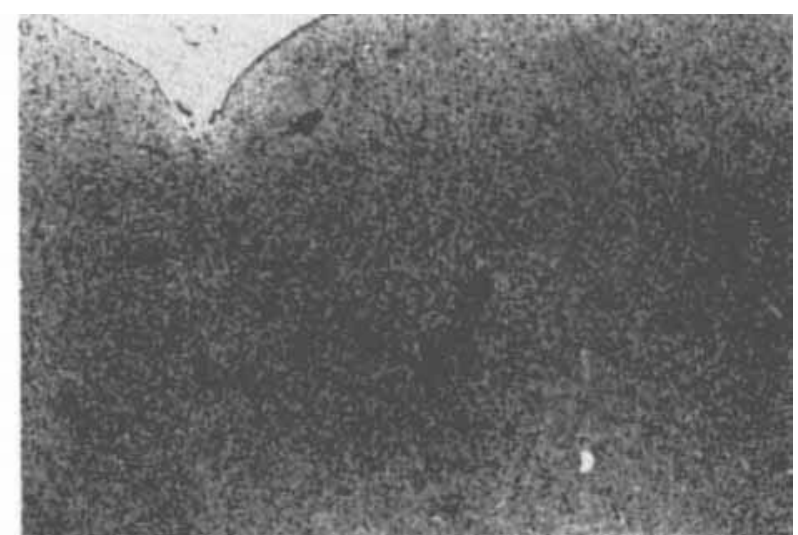

B

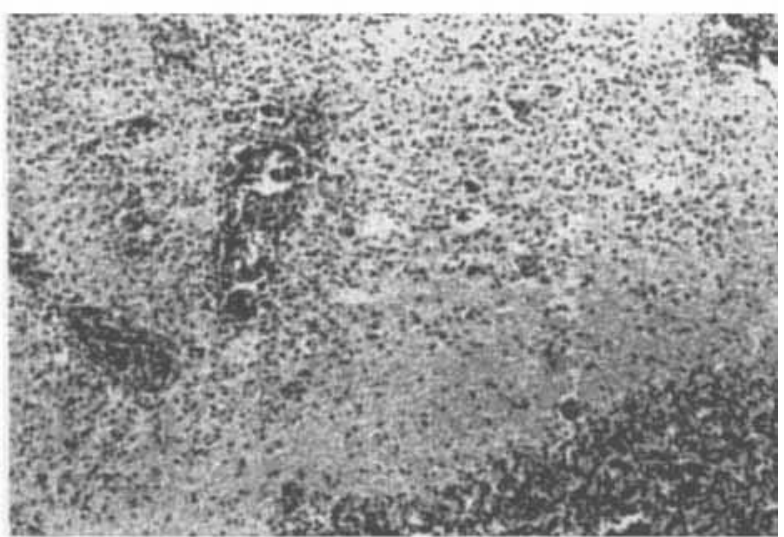

D

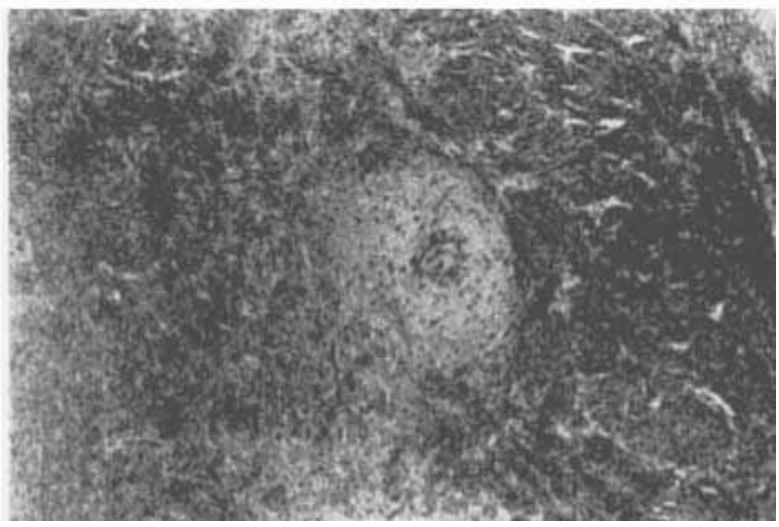

F

flammatory cuffs. (HEE, $\times 133$ before $35 \%$ reduction.) (D) $A$ bigher-power view of the lesion edge. $(\times 250$ before $35 \%$ reduction.) (E) Animal 183-88. Lesion in corona radiata comprised of perivascular mononuclear cell inflammation and foamy macrophages; reactive astrocytes and demyelination (pale perivascular zone) can be identified. (HEE, $\times 400$ before $35 \%$ reduction.) (F) Animal 130-88. External capsule. Perivenous inflammation, demyelination, and astrogliosis. (Luxol fast bluelperiodic acid-Schiff stain, $\times 200$ before $36 \%$ reduction.) 
PBMC

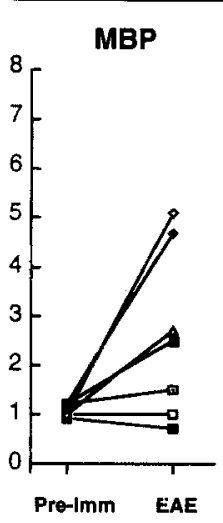

BH

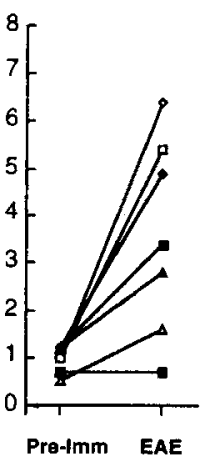

PLP
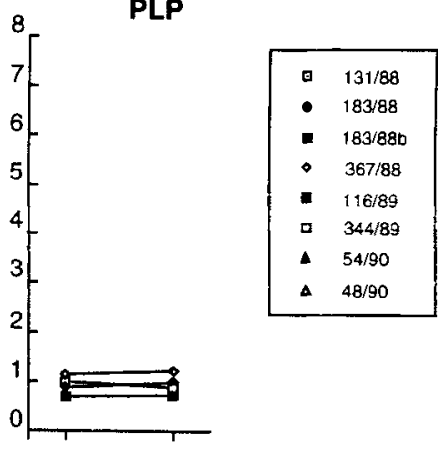

Pre-Imm EAE

\section{SPLEEN CELLS}

MBP

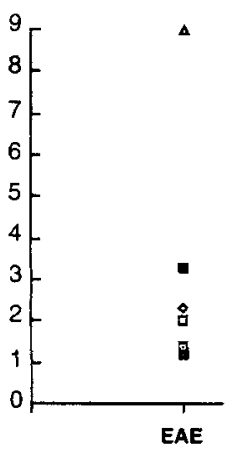

BH

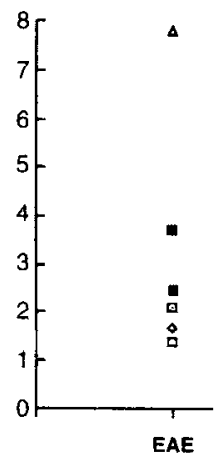

PLP

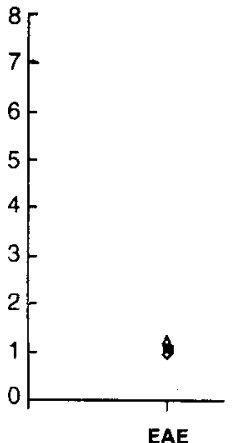

\section{LYMPH NODE CELLS}

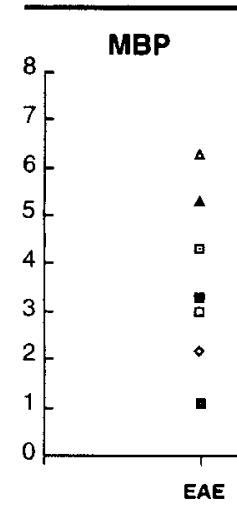

BH

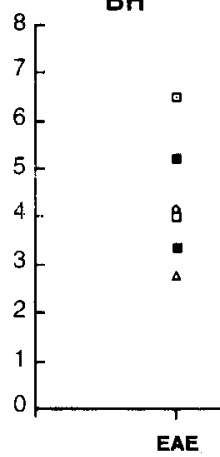

PLP

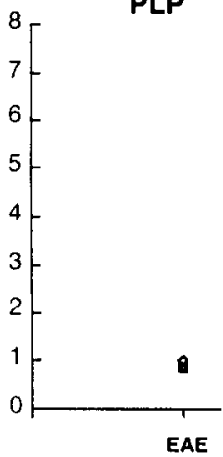

Fig 5. T-lymphocyte reactivity to brain antigens during the course of experimental autoimmune encephalomyelitis (EAE). Data are expressed as stimulation indices (SIs) to $M B P, B H$, and PLP. For peripberal blood mononuclear cells (PBMCs), SIs are shown at baseline before immunization and again following development of dinical signs of EAE. For spleen and lympb node cells, preimmunization SIs were not obtained. In PBMCs, spleen, and lymph nodes, all EAE time point studies were per- formed from cells bavested at the time of death corresponding to the following number of days after the initial appearance of clinical signs: Animal 1.31-88 = 3 days; Animal 183-88a $=5$ days: Animal 183-886 = 5 days; Animal 116-89 = 7 days; Animal 344-89 = 26 days; Animal 54-90 = 2 days; and Animal $48-90=2$ days. $M B P=$ myelin basic protein; $B H=$ brain white matter bomogenate; $P L P=$ proteolipid protein. 

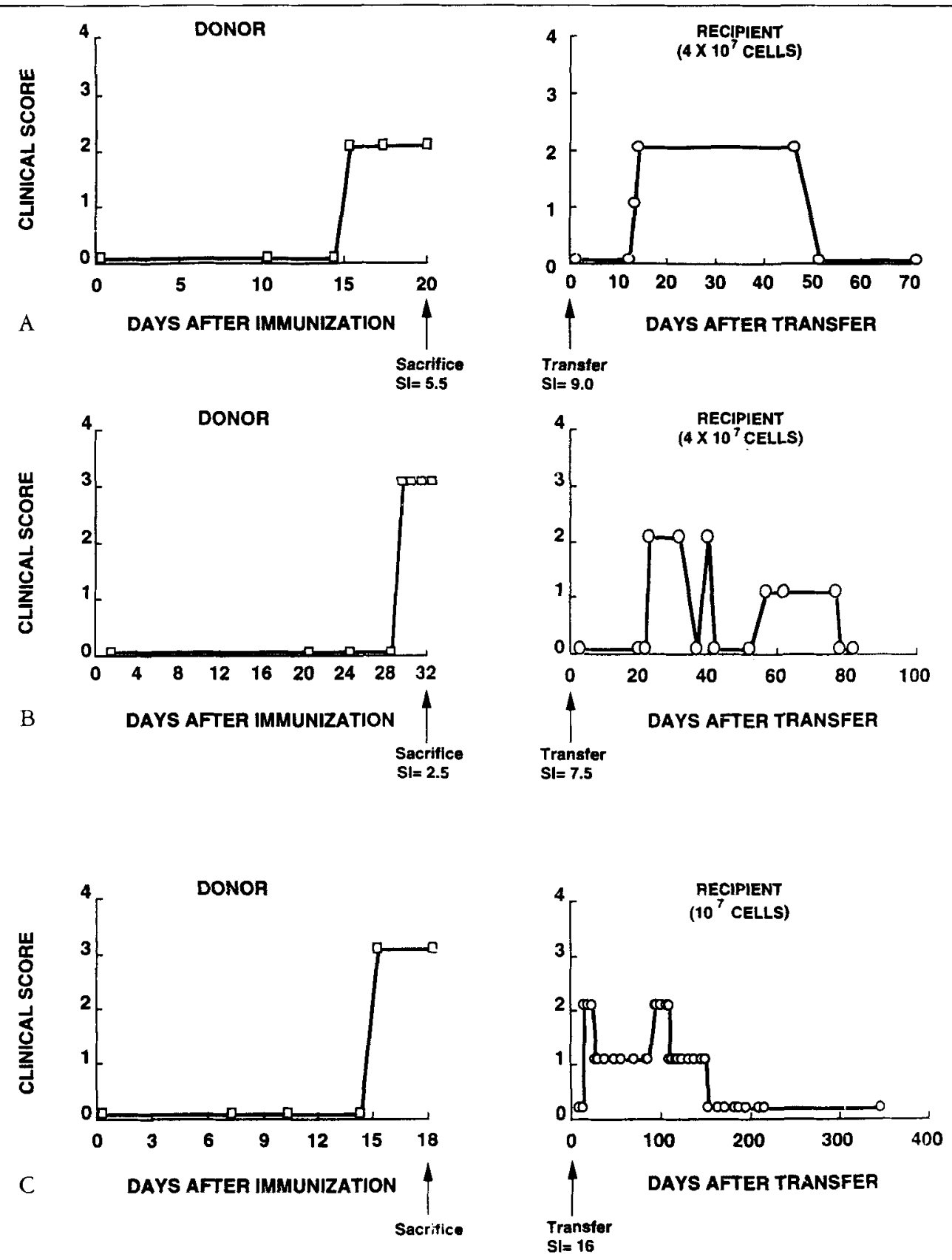

Fig 6. Clinical course characteristics of adoptive transfer experimental autoimmune encephalomyelitis (EAE) mediated by myelin basic protein $(M B P)$-reactive $T$ cells in $\mathrm{C}$. jacchus. $(A-C)$ Three independent transfers are summarized. Donor animals (left) were actively immunized, and at the onset of EAE spleen and lymph node cells were barvested. Following expansion, each $T$-cell line $(A, B$, or $C)$ was adoptively transferred into a different chimeric recipient (right). Stimulation indices (SI) of wbole splenic and lymph node cells at the time of death, and of each transferred line at transfer, are noted. 
tain significance was identified. The paucity of postmortem changes were in marked contrast to the widespread abnormalities present in the active immunization EAE group.

Following expansion in vitro, each of the three lines capable of mediating EAE by adoptive transfer recognized a different region of the MBP molecule-aa 11 to 30,31 to 50 , and 153 to 172 , respectively. These data suggest that the repertoire of MBP-reactive $\mathrm{T}$ cells in $C$. jacchus may be diverse, and that multiple epitopes may be capable of stimulating an encephalitogenic response in this species.

\section{Discussion}

In this report we describe a nonhuman primate model of EAE that is characterized clinically by mild neurological signs and a chronic course, and parhologically by primary CNS demyelination. In the past, EAE has been extensively studied in primates, theoretically an ideal model system for MS because of the genetic similarities between human and nonhuman primates. However, in these animals a chronic relapsing course is not reliably observed $[20,21\}$ and the hyperacute necrotichemorrhagic CNS lesions that are characteristic of acute EAE differ substantially from the typical pathology of human demyelinating diseases.

In earlier experiments that employed different immunization regimens, $C$. jacchus was found to be resistant to actively induced EAE (data not shown). Intravenous administration of Bordetella pertussis vaccine in combination with white matter in adjuvant was required for active induction of disease. This requirement for pertussis vaccine distinguishes $\mathrm{EAE}$ in C. jacchus from other nonhuman primate forms of EAE, although in other species, for example, the $\mathrm{H}-2^{\mathrm{S}}$ mouse, a similar immunization strategy is required [3]. In C. jaccbus, Bordetella pertussis may function as an adjuvant or as a modifier of blood-brain barrier integrity [33, 34]; it does not by itself induce disease [35]. Clinical features of EAE in C. jacchus, notably a chronic relapsing-remitting course, mild neurological signs (mean clinical score of 2), and complete recovery from the initial attack, distinguish it from other primate models of EAE. In macaques, clinical scores of 4 or higher are common during the initial attack and are associated with fatal outcome $[20,21]$. Furthermore, induction of chronic relapsing $\mathrm{EAE}$ in macaques has been difficult. In rhesus macaques, induction of chronic disease generally requires steroid or other treatment administered at the time of acute disease, interventions that limit the usefulness of this model [20]. In cynomolgus monkeys, a chronic course may occur without administration of immunosuppressive drugs, yet approximately half of immunized animals die from the acute disease [21].
Acute EAE lesions in C. jacchus were characterized by scattered perivascular inflammatory infiltrates surrounded by large concentric areas of demyelination and associated with intense macrophage infiltration and mild astrogliosis. Inflammation was largely restricted to white matter in brain and spinal cord. The prominent demyelination, and the relatively sparse mononuclear cell infiltration, are the major distinguishing features of acute $\mathrm{EAE}$ in C. jacchus. By contrast, inflammation, rather than demyelination, is the predominant feature of acute EAE in rodents. In the Lewis rat, for example, lesions predominate in the spinal cord and involve gray as well as white matter, and no demyelination is present [10]. Similarly, in the guinea pig and mouse, limited or scant demyelination occurs in acute EAE [1-7, 9]. In larger species, for example, rabbits and macaque monkeys, demyelination is observed in acute EAE but hemorrhagic and necrotic lesions and polymorphonuclear cell infiltration also occur [19-21].

Compared with acute lesions, chronic lesions contained denser and more widely scattered mononuclear cell infiltration, and more prominent gliosis. This was particularly evident in the spinal cord. Chronic forms of EAE have been previously described in rats [8], mice $[7,9]$, guinea pigs [4], and macaques $[20,21]$, models that are in general associated with more prominent demyelination than is present in acute EAE. In C. jacchus, the sharply demarcated areas of demyelination and gliosis concentric to the perivascular infiltrates indicate the formation of plaques, a feature consistently present in this model.

The natural bone marrow chimerism of $C$. jacchus permitted the adoptive transfer of neurological signs of EAE by MBP-reactive T-cell lines. Transfer was successful with two MBP-reactive short-term lines, and with one of two MBP-reactive long-term lines. The failure of one long-term line to transfer EAE was not due to a difference in its reactivity to MBP measured by its SI. The nonencephalitogenic long-term line recognized aa 92 to 111 of MBP, a fragment not recognized by any encephalitogenic line in this study. It is possible that the nonencephalitogenicity is due to recognition of this particular region of MBP. However, in rodent EAE models, it has been observed that some MBP-reactive clones recognize encephalitogenic fragments of MBP but fail to transfer EAE because of their pattern of cytokine secretion [36]. In C. jacchus, we found that most MBP-reactive T-cell clones derived from peripheral blood do induce EAE by adoptive transfer [35]. Current work will define whether T-cell phenotypes defined by their cytokine profiles are predictive of encephalitogenicity in marmosets.

Adoptive transfer of MBP-reactive T-cell lines resulted in clinical signs of EAE, but no demyelination was evident at autopsy. We also found that active im- 
munization with purified MBP induces inflammatory changes in the meninges and peripheral nerve roots, but little or no demyelination (C. Genain, unpublished data, 1995). This lack of demyelination contrasts sharply with the large demyelinating lesions that follow immunization with whole white matter. In addition to MBP, other myelin proteins may also be encephalitogenic in C. jacchus, as is the case for rodents. These include PLP [37] and myelin oligodendrocyte glycoprotein [38], which may induce demyelinating forms of EAE. Although in this report proliferative responses to PLP could not be detected, it is likely that a complex immune response to more than one white matter antigen underlies the primary demyelinating pathology in C. jacchus CNS. This response may involve not only $\mathrm{T}$ cells but antibody production by $\mathrm{B}$ cells as well [39].

Mapping data from three different encephalitogenic lines suggested that $\mathrm{T}$-cell recognition of diverse epitopes of MBP could result in clinical disease, although the possibility that a common, undetected epitope was recognized by all lines could not be excluded. Additional data, however, employing MBP-reactive T-cell clones obtained from the circulation established conclusively that the T-cell repertoire to MBP is diverse in C. jacchus [35], as also appears to be the case in humans $[40]$.

In summary, the data presented here indicate that a demyelinating form of acute EAE can be consistently induced in the primate species $C$. jaccbus. To our knowledge, there exist no comparable examples of acute EAE lesions that mimic those of acute MS. Chronic lesions indistinguishable from lesions of chronic active MS are also described. A final advantage of the $C$. jaccbus model is the natural bone marrow chimerism that exists between siblings and that makes possible the adoptive transfer of EAE by MBP-reactive T-cell lines. This should permit dissection of the immunological mechanisms that mediate autoimmune demyelination in primates.

These studies were supported by the National Institutes of Health (RR 00168 and NS 30727), and by grants from the Penates Foundation and Devenshire Trust. Human white matter was provided by the Brain Tissue Resource Center, which is supported in part by Public Health Service grant MH/NS 31862. Luca Massacesi was a fellow of the Italian Multiple Sclerosis Society. Claude P. Genain is a postdoctoral fellow from the National Multiple Sclerosis Society.

The authors with to thank Dr Marjorie B. Lees who generously provided proteolipid apoprotein for these studies. The authors also wish to thank Shelley Ambrose, Leslie Hopper, and Laurie McCarthy for technical assistance, and Caroline Figoni for secretarial assistance.

\section{References}

1. Alvord EC, Kies MW, Suckling AJ. Experimental allergic encephalomyelitis: a useful model for multiple sclerosis. Prog Clin Biol Res 1984;146:1-554
2. Raine CS. Biology of disease: the analysis of autoimmune demyelination: irs impact on multiple sclerosis. Lab Invest 1984;50: 608-635

3. Bernard CCA, Carnegie PA. Experimental autoimmune encephalitis in mice: immunologic responses to mouse spinal cord and myelin basic proteins. J Immunol 1975;114:1537

4. Alvord EC Jr, Driscoll BF, Kies MW. Large subpial plaques of demyelination in a new form of chronic experimental allergic encephalomyelitis in the guinea pig. Neurochem Pathol 1985;3: $195-214$

5. Paterson PY. Transfer of allergic encephalomyelitis in rats by means of lymph node cells. J Exp Med 1960;111:119-135

6. Pettinelli DB, McFarlin DE. Adoptive transfer of experimental allergic encephalomyelitis in SJL/J mice after in vitro activation of lymph node cells by myelin basic protein: requirement for Lyt 1 + 2 - T-lymphocytes. J Immunol 1981;127:1420-1423

7. Zamvil S, Nelson P, Trotter J, et al. T-cell clones specific for myelin basic protein induce chronic relapsing paralysis and demyelination. Nature 1985;317:355-358

8. McFarlin DE, Blank SE, Kibler RF. Recurrent experimental allergic encephalomyelitis in the Lewis rat. J Immunol 1974;1 I3 712

9. Raine CS, Mokatarian F, McFarlin DE. Adoptively transferred chronic relapsing experimental autoimmune encephalomyelitis in the mouse: neuropathologic analysis. Lab Invest 1984;5:534546

10. Stohl W, Gonatas NK. Chronic permeability of the central nervous system to mononuclear cells in experimental allergic encephalomyelitis in the Lewis rat. J Immunol 1978;120:844

11. Blankenhorn EP, Stranford SA. Genetic factors in demyelinating diseases: genes that control demyelination due to experimental allergic encephalomyelitis (EAE) and Theiler's murine encephalitis virus. Reg Immunol 1992;4:331-343

12. Goverman J, Woods A, Larson L, et al. Transgenic mice that express a myelin basic protein-specific $T$ cell receptor develop spontaneous autoimmunity. Cell 1993;72:551-560

13. Kumar V, Sercarz EE. The involvement of $T$ cell receptor peptide-specific regulatory $\mathrm{CD} 4+\mathrm{T}$ cells in recovery from antigen-induced autoimmune disease. J Exp Med 1993;178:909916

14. Zamvil SS, Steinman L. The T-lymphocyte in experimental allergic encephalomyelitis. Annu Rev Immunol 1990;8:579-621

15. Hickey WF, Hsu BL, Kimura $\mathrm{H}$. T lymphocyte entry into the central nervous system. J Neurosci Res 1991;28:254-260

16. Rivers TM, Schwenther FF. Encephalomyelitis accompanied by myelin destruction experimentally produced in monkeys. J Exp Med 1935;61:689-702

17. Ferraro A, Cazzullo CL. Chronic experimental allergic encephalomyelitis in monkeys. J Neuropathol Exp Neurol 1948;7:235260

18. Ravkina L, Rogova Y, Lazarenko L. Chronic experimental allergic encephalomyelitis in rhesus monkeys and its modification by treatment. J Neurol Sci 1978;38:281-293

19. Alvord EC Jr, Shaw CM, Hruby S. Myelin basic protein treatment of experimental allergic encephalomyelitis in monkeys Ann Neurol 1979;6:467-473

20. Shaw CM, Alvord EC Jr, Hruby S. Chronic remitting-relapsing experimental allergic encephalomyelitis induced in monkeys with homologous myelin basic protein. Ann Neurol 1988;24: 738-748

21. Massacesi L, Joshi N, Lee-Parritz D, et al. Experimental allergic encephalomyelitis in cynomolgus monkeys: quantitation of $\mathrm{T}$ cell response. J Clin Invest 1992;90:399-404

22. Kibler RF, Re PK, McKneally S, Shapira R. Biological activity of an encephalitogenic fragment in the monkey. J Biol Chem $1972 ; 2: 969-972$ 
23. Eylar EH, Brostoff S, Jackson J, Carter H. Allergic encephalomyelitis in monkeys induced by a peptide from the $A I$ protein. Proc Natl Acad Sci USA 1972;69:617-619

24. Karkhanis YD, Carlo DJ, Brostoff SW, Eylar EH. Allergic encephalomyelitis. J Biol Chem 1975;5:1718-1722

25. Picus JW, Aldrich R, Letvin NL. A naturally occurring bone marrow chimeric primate. Transplantation 1985;39:297-301

26. Deibler GE, Martenson RE, Kies MW. Large scale preparation of myelin basic protein in central nervous tissue of several mammalian species. Prep Biochem 1972;2:139-165

27. Folch J, Lees MB, Sloane Stanley GH. A simple method for isolation and purification of total lipids from animal tissues. J Biol Chem 1957;266:497-509

28. Bizzozero O, Besio-Moreno M, Pasquini JM, et al. Rapid purification of proteolipids from rat subcellular fractions by chromatography on a lipophilic dextran gel. J Chromatogr 1982;27: $33-44$

29. Lees MB, Sakura JD. In: Marks N, Rodnight R, eds. Research methods in neurochemistry, preparation of proteolipids, vol 4 New York: Plenum, 1979:354-370

30. Tabira J, Kira JI. Strain and species differences of encephalitogenic determinants of myelin basic protein and proteolipid apoprotein. In: Martenson RE, ed. Myelin: biology and chemistry. Ann Arbor, MI: CRC Press, 1992:783-799

31. Welch A, Holda JH, Swanborg RH. Regulation of experimental allergic encephalomyelitis. II. Appearance of suppressor cells during the remission phase of the disease. J Immunol 1980;125: 186-189

32. MacPhee IAM, Mason DW. Studies of refractoriness to reinduction of experimental allergic encephalomyelitis in Lewis rats that have recoveted from one episode of the disease. J Neuroimmunol 1990;27:9-19

33. Linthicum DS, Mugnoz JJ, Blaskett A. Acute experimental aller- gic encephalomyelitis in mice. The adjuvant action of Bordetella pertussis is due to vasoactive amine sensitization and increased vascular permeability of the central nervous system. Cell Immunol 1982;73:299-310

34. Sudweeks JD, Todd JA, Blankenhorn EP, et al. Locus controlling Bordetella pertussis-induced histamine sensitization (Bphs), an autoimmune disease-susceptibility gene, maps distal to T-cell receptor $\beta$-chain gene on mouse chromosome 6. Proc Natl Acad Sci USA 1993;90:3700-3704

35. Genain CP, Parritz DL, Nguyen MH, et al. In healthy primates, circulating autoreactive T-cells mediate autoimmune disease. J Clin Invest 1994;94:1339-1345

36. Powell MB, Mitchell D, Lederman J, et al. Lymphotoxin and tumor necrosis factor-alpha productions by myelin basic proteinspecific T-cell clones correlates with encephalitogenicity. Int Immunol 1990;2:539-544

37. Tuohy VK, Sobel RA, Lees MB. Myelin proteolipid proteininduced experimental allergic encephalomyelitis; variations of disease expression in different strains of mice. J Immunol 1988; 140:1868

38. Linington $C$, Berger $T$, Perry $L$, et al. $T$ cells specific for the myelin oligodendrocyte glycoprotein, mediate an unusual autoimmune inflammatory response in the central nervous system. Eur J Immunol 1993;23:1364-1373

39. Lassmann $H$, Brunner $C$, Bradl $M$, Linington $C$. Experimental allergic encephalomyelitis: the balance between encephalitogenic $T$ lymphocytes and demyelinating antibodies determines size and structure of demyelinated lesions. Acta Neuropathol (Berl) 1988;75:566-576

40. Joshi N, Usuku K, Hauser SL. The T-cell response to myelin basic protein in familial multiple sclerosis: diversity of fine specificity restricting elements, and T-cell receptor usage. Ann Neurol 1993;34:385-393 